\title{
Reservoir Multiscale Data Assimilation Using the Ensemble Kalman Filter
}

\author{
Santha R. Akella \\ Department of Earth and Planetary Sciences, Johns Hopkins University, Baltimore, USA \\ E-mail: santha.akella@jhu.edu \\ Received June 22, 2010; revised November 11, 2010; accepted November 15, 2010
}

\begin{abstract}
In this paper we propose a way to integrate data at different spatial scales using the ensemble Kalman filter (EnKF), such that the finest scale data is sequentially estimated, subject to the available data at the coarse scale (s), as an additional constraint. Relationship between various scales has been modeled via upscaling techniques. The proposed coarse-scale EnKF algorithm is recursive and easily implementable. Our numerical results with the coarse-scale data provide improved fine-scale field estimates when compared to the results with regular EnKF (which did not incorporate the coarse-scale data). We also tested our algorithm with various precisions of the coarse-scale data to account for the inexact relationship between the fine and coarse scale data. As expected, the results show that higher precision in the coarse-scale data, yielded improved estimates.
\end{abstract}

Keywords: Kalman Filter, Reservoir Engineering, Uncertainty Quantification, Multiscale Data

\section{Introduction}

The principal objective of data assimilation methods [1] is to combine the information provided by measured data and a (numerical) forecast model to obtain an improved estimate of the system state (and parameters). Unlike variational methods which require availability of complex adjoint models for data assimilation, the ensemble Kalman filter (EnKF) can be quickly implemented and one can also obtain uncertainty estimates via error variancecovariance propagation; see [2] and references therein for further details. The EnKF is a sequential Monte Carlo method based on Bayes theorem. The method is increasingly being used for estimating unknown model state and parameters in various geological and hydrological models [3].

One of the major problems in subsurface characterization is the huge uncertainty in the knowledge of hydrocarbon reservoir permeability and porosity. Since the flow of hydrocarbons such as oil and gas through the subsurface formation critically depends on the geological rock properties, it is important to accurately know these properties. This article focuses on methods to obtain more accurate quantification of the reservoir rock properties using measured data. Broadly speaking, the measured data used for description of reservoir porosity and permeability characterization consist of static and dynamic data. Static data such as well logs, core samples can resolve heterogeneity at a scale of a few inches or feet with high reliability. However, dynamic data such as fractional flow (defined as the ratio of the injection fluid to the total fluid produced at the production wells; or water cut), pressure transient and tracer test data typically scan the length scales comparable to the inter-well distances. Additional dynamic data such as time-lapse seismic images [4] can provide improved spatial sampling, but at a lower precision. A majority of previous studies on uncertainty quantification in reservoir performance forecasting using EnKF have mostly dealt with integration of dynamic data (for e.g., [5-7]). However it is widely recognized that integration of additional multiscale data could further reduce the uncertainty (see $[8,9]$ and references therein). With that perspective, integration of data at coarse-and fine-scales, is an important objective and is addressed in this paper. We use the EnKF to estimate fine-scale fields for subsurface characterization. Also, our method could be generalized to other sequential data assimilation methods such as particle filtering (where, rather than updating the ensemble members model state, we update the probability assigned to each ensemble member based on model data misfit). The main reason why we used EnKF in this paper is because it requires 
fewer ensemble members than the particle filters, see [3] and references therein for further details.

In this paper, apart from the water cut data, we consider coarse-scale measured data as well. The coarse-scale data is assumed to be permeability at some specified level of precision. The unknown variables: permeability, at the fine-scale, are estimated using a modification to the EnKF algorithm, linking the data at different scales via upscaling. It is important to resolve fine-scale heterogeneity for various purposes such as, enhanced oil recovery, environmental remediation, etc. The main idea behind upscaling is to obtain an effective coarse-scale permeability which yields the same average response as that of the underlying fine-scale field, locally. Single phase flow upscaling procedures for two phase flow problem have been discussed by many authors; see e.g., $[10,11]$ and also Section 3.1. We will refer to our proposed variant of EnKF as coarse-scale EnKF. Assimilation using dynamic data, such as fractional flow data only, is therefore referred to as regular EnKF. The coarse-scale permeability data could be obtained either from geologic consideration or coarse-scale inversion of dynamic, fractional flow data on a coarse grid as considered in $[8,12]$. This coarse-scale, static data can be viewed as a constraint, which is to be satisfied up to the prescribed variance for obtaining the fine-scale estimates in every data assimilation cycle. Upscaling methods relate the solution at the finescale to the coarse-scale, therefore in the Kalman filter context, it amounts to modeling a nonlinear observation operator. In our coarse-scale EnKF approach, we use the measured data in batches, such that the estimate with one data becomes a prior while assimilating the other observation (see Section 3 for further details). Though in this paper we used coarse-scale data at only one scale, our approach can be easily generalized to assimilate data at multiple scales by appropriately modeling the linkage between different scales. Also, our upscaling method is independent of the underlying finescale field.

For the purpose of self-contendness and notational clarity, we briefly review the governing equations, sequential data assimilation using the ensemble Kalman filter in Section 2, which is followed by a description of the coarse-scale EnKF algorithm (Section 3). For our numerical results (Section 4), we consider a five-spot pattern, with the injection well placed in the middle of a rectangular domain and four production wells located at the vertices of the rectangle. A reference case is used to provide true data, which is randomly perturbed to obtain synthetic measurements. A comparison of the regular EnKF with the coarse-scale EnKF (Sections 4.1 and 4.2 respectively) shows that using coarse-scale permeability data (via coarse-scale EnKF) significantly improves the fine-scale estimates as well as future fractional flow prediction.

\section{Background}

\subsection{Fine-Scale Model}

In this paper, we consider two-phase flow in a subsurface formation under the assumption that the displacement is dominated by viscous effects. For simplicity, we neglect the effects of gravity, compressibility, and capillary pressure, although our proposed approach is independent of the choice of physical mechanisms. Also, porosity will be considered to be constant. The two phases will be referred to as water and oil (or a non-aqueous phase liquid), designated by subscripts $w$ and $o$, respectively. We write Darcy's law for each phase as follows:

$$
\begin{gathered}
v_{j}=-\frac{k_{r j}(S)}{\mu_{j}} \kappa_{f} \nabla p r ; \nabla \cdot\left(\lambda(S) \kappa_{f} \nabla p r\right)=h, \\
\lambda(S)=\frac{k_{r w}(S)}{\mu_{w}}+\frac{k_{r o}(S)}{\mu_{o}}, f(S)=\frac{k_{r w}(S) / \mu_{w}}{k_{r w}(S) / \mu_{w}+k_{r o}(S) / \mu_{o}}, \\
v=v_{w}+v_{o}=-\lambda(S) \kappa_{f} \cdot \nabla p r ; \phi \frac{\partial S}{\partial t}+v \cdot \nabla S
\end{gathered}
$$

The above descriptions are referred to as the fine-scale model of the two-phase flow problem. Here $\kappa_{f}$ is the (fine-scale) permeability of the medium, $\lambda(S)$ is the total mobility, $\mu_{j}$ denotes phase viscosity, $p r$ is the pressure, $h$ is the source term, $\phi$ and $S$ denote porosity and water saturation (volume fraction), respectively.

\subsection{Sequential Estimation using EnKF}

Using dynamic measured data such as water cut, we can sequentially estimate the unknown parameters (permeability, porosity, etc.) and state variables such as pressure, water saturation (two-phase flow) and production data at well locations using the EnKF as discussed in [5,7,13]. Following these previous works, in this paper we assume that the only dynamic data available is water cut data, and that porosity is known. The combined state-parameter to be estimated are given by $\Psi=\left[\ln \left(\kappa_{f}\right), \mathbf{p r}, \mathbf{S}, \mathbf{W}_{c}\right]^{T}$. Where $\ln (\cdot)$ is natural logarithm of permeability field and $\mathbf{W}_{c}$ denotes water cut; in order to distinguish observed water cut from model predicted water cut, now onwards we will denote the observed water cut data $\mathbf{W}_{c}^{o}$, by $\mathbf{y}$.

The EnKF introduced in [14] is a sequential Monte Carlo method where an ensemble of model states evolve in state-space with mean as the best estimate and spread of the ensemble as the error covariance, as summarized in the following steps. Each of the ensemble members is 
forecasted independently (in this work, we neglected modeling errors),

$$
\mathbf{\Psi}_{n+1}^{(i)}=F\left[\mathbf{\Psi}_{n}^{(i)}\right],
$$

where $F[\cdot]$ is the forecast operator (Equations (1), (2)), superscript $(i)$ denotes the $i^{\text {th }}$ ensemble member; now onwards we will drop the time subscript. The ensemble mean and covariance are defined as,

$$
\begin{gathered}
\overline{\boldsymbol{\Psi}}=\frac{1}{N_{e}} \sum_{i=1}^{N_{e}} \boldsymbol{\Psi}^{(i)}, \\
\mathbf{P}^{f} \approx \frac{1}{N_{e}-1} \mathbf{A}^{\prime}\left(\mathbf{A}^{\prime}\right)^{T},
\end{gathered}
$$

where $\quad \mathbf{A}^{\prime}=\left(\mathbf{b}^{(1)}, \mathbf{b}^{(2)}, \cdots, \mathbf{b}^{\left(N_{e}\right)}\right), \quad \mathbf{b}^{(i)}=\boldsymbol{\Psi}^{(i)}-\overline{\boldsymbol{\Psi}}, \quad$ and $N_{e}$ is the number of ensemble members. The observation vector for each ensemble member is given by,

$$
\mathbf{y}^{(i)}=H\left[\mathbf{\Psi}^{t}\right]+v^{(i)},
$$

where $\mathbf{H}\left[\boldsymbol{\Psi}^{t}\right]$ is the observed data from the truth and $v^{(i)}$ represents observational errors, which are i.i.d. samples [15] from a normal distribution with zero mean and variance, $\mathbf{R}$. We note that if only the water cut data is being measured, the mapping from model-to-observational space, $\mathbf{H}$ is trivially equal to $[\mathbf{0 0 0} \mathbf{I}]$, since $\boldsymbol{\Psi}=\left[\ln (\kappa), \mathbf{p r}, \mathbf{S}, \mathbf{W}_{c}\right]^{T}$.

The forecasted ensemble (Equation (3)) is updated by assimilating the observed data,

$$
\widetilde{\boldsymbol{\Psi}}^{(i)}=\boldsymbol{\Psi}^{(i)}+\mathbf{K}\left(\mathbf{y}^{(i)}-\mathbf{H}\left[\boldsymbol{\Psi}^{(i)}\right]\right),
$$

where $\mathbf{K}$ is the Kalman gain, given by

$$
\mathbf{K}=\mathbf{P}^{f} \mathbf{H}^{T}\left[\mathbf{H} \mathbf{P}^{f} \mathbf{H}^{T}+\mathbf{R}\right]^{-1} .
$$

Computationally efficient implementation of the EnKF is discussed for e.g., in [2,16]. We use the above set of corrected ensemble states, $\left\{\widetilde{\boldsymbol{\Psi}}^{(i)}\right\}_{i=0}^{N_{e}}$ in the simulation model (Equation (3)) to predict until the next set of observational data is available.

\section{Coarse-Scale Constrained EnKF}

The EnKF presented so far, used only the dynamic, production data (water cut) $\mathbf{y}$, with error $v=\mathbf{y}-\mathbf{H}\left[\boldsymbol{\Psi}^{t}\right]$, $v \sim \mathcal{N}(\mathbf{0}, \mathbf{R})$ to update the ensemble (Equation (7)). In addition to $\mathbf{y}$, if we are also given static data (as mentioned in the Introduction), which is another set of independently measured data, z. Assuming that the corresponding measurement error is given by $\omega=\mathbf{z}-\mathbf{U}\left[\boldsymbol{\Psi}^{t}\right], \quad \omega \sim \mathcal{N}(\mathbf{0}, \mathbf{Q}) ; \quad \mathbf{U}: \boldsymbol{\Psi} \mapsto \mathbf{z}$. Its likelihood is given by

$$
p(\mathbf{z} \mid \boldsymbol{\Psi}) \propto \exp \underbrace{\left\{-\frac{1}{2}(\mathbf{z}-\mathbf{U}[\boldsymbol{\Psi}])^{T} \mathbf{Q}^{-1}(\mathbf{z}-\mathbf{U}[\boldsymbol{\Psi}])\right\}}_{\mathcal{J}_{z}} .
$$

If this static data $\mathbf{z}$, corresponds to coarse-scale permeability data $[12,8]$, then $\mathbf{U}=[\mathcal{U} \mathbf{0 0 0}]$. Where $\mathcal{U}: \kappa_{f} \mapsto \kappa_{c}$, is a nonlinear mapping that maps the fine-scale permeability field $\left(\kappa_{f}\right)$ to coarse-scale field $\left(\kappa_{c}\right)$ via an upscaling procedure (e.g., $[17,18])$, details are provided in Section 3.1. Note that by definition, the errors in water-cut data, $\mathbf{y}$ and coarse-scale permeability, $\kappa_{c}$ data don't influence each other, since they are entirely differently measured quantities.

Now, our goal is to obtain an estimate which is based on both of the above dynamic and static data. The likelihood of $\mathbf{y}$ is given by

$$
p(\mathbf{y} \mid \mathbf{\Psi}) \propto \exp \underbrace{\left\{-\frac{1}{2}(\mathbf{y}-\mathbf{H}[\mathbf{\Psi}])^{T} \mathbf{R}^{-1}(\mathbf{y}-\mathbf{H}[\mathbf{\Psi}])\right\} .}_{\mathcal{J}_{y}}
$$

The probability distribution function (pdf) of the predicted ensemble,

$$
p(\boldsymbol{\Psi}) \propto \exp \underbrace{\left\{-\frac{1}{2}(\boldsymbol{\Psi}-\overline{\boldsymbol{\Psi}})^{T}\left(\mathbf{P}^{f}\right)^{-1}(\boldsymbol{\Psi}-\overline{\boldsymbol{\Psi}})\right\}}_{\mathcal{J}_{f}},
$$

where $\overline{\boldsymbol{\Psi}}$ and $\mathbf{P}^{f}$ are the predicted ensemble mean and covariance respectively (Equations (4) and (5)). Then, using Bayes theorem, we obtain

$$
\begin{aligned}
p(\boldsymbol{\Psi} \mid \mathbf{z}, \mathbf{y}) & =\frac{p(\boldsymbol{\Psi}, \mathbf{z}, \mathbf{y})}{p(\mathbf{z}, \mathbf{y})} \\
& =\frac{p(\mathbf{z}, \mathbf{y} \mid \mathbf{\Psi}) p(\boldsymbol{\Psi})}{p(\mathbf{z}, \mathbf{y})} \propto p(\mathbf{z}, \mathbf{y} \mid \Psi) p(\boldsymbol{\Psi}) \\
& =p(\mathbf{z} \mid \boldsymbol{\Psi}) \underbrace{p(\mathbf{y} \mid \boldsymbol{\Psi}) p(\boldsymbol{\Psi}) .}_{\propto p(\boldsymbol{\Psi} \mid \mathbf{y})}
\end{aligned}
$$

The last term in above equation implies that the two independent data, $\mathbf{y}$ and $\mathbf{z}$ can be sequentially assimilated in the following two steps. We first assimilate observation $\mathbf{y}$ to obtain an intermediate ensemble, $\left\{\widetilde{\boldsymbol{\Psi}}^{(i)}\right\}_{i=0}^{N_{e}}$ as discussed in Section 2.2.

$$
p(\widetilde{\boldsymbol{\Psi}})=p(\boldsymbol{\Psi} \mid y) \propto \exp \left\{-\left(\mathcal{J}_{f}+\mathcal{J}_{y}\right)\right\},
$$

This intermediate ensemble and likelihood in Equation (8), can then be combined to obtain the final estimate

$$
\begin{aligned}
& \left\{\widehat{\boldsymbol{\Psi}}^{(i)}\right\}_{i=0}^{N_{e}} \cdot \\
& \quad p(\widehat{\boldsymbol{\Psi}})=p(\boldsymbol{\Psi} \mid \mathbf{z}, \mathbf{y}) \propto \exp \left\{-\left(\mathcal{J}_{f}+\mathcal{J}_{y}+\mathcal{J}_{z}\right)\right\},
\end{aligned}
$$

Therefore, in a least-squared sense, the final estimate maximizes the posterior pdf $p(\Psi \mid z, y)$ which corres- 
ponds to the minimum of $\mathcal{J}=\mathcal{J}_{z}+\mathcal{J}_{y}+\mathcal{J}_{f}$. See Appendix A, for further details (where we show that the solution $\widehat{\boldsymbol{\Psi}}^{(i)}$ corresponds to the minimum of $\mathcal{J}$, for any $i^{\text {th }}$ ensemble member). The coarse-scale EnKF algorithm is detailed in Appendix B.

\subsection{Upscaling Methods}

In brief, the main idea behind upscaling of absolute fine-scale permeability is to obtain effective coarse-scale permeability for each coarse-grid block. Once the upscaled absolute permeability is computed, the original equations are solved on the coarse-grid, without changing the form of relative permeability curves. This is an inexpensive calculation, since the pressure update involves only solving the pressure equation on the coarse-grid, and one can take larger time step for solving the transport equation. In our numerical simulations, the fine-grid is coarsened 10 times in each direction. These kinds of upscaling techniques in conjunction with the upscaling of absolute permeability have been used in groundwater applications (see e.g., [18]).

The link between the coarse and the fine-scale permeability fields is usually nontrivial because one needs to take into account the effects of all the scales present at the fine level. In the past simple arithmetic, harmonic or power averages have been used to link properties at various scales. These averages can be reasonable for low heterogeneities or for volumetric properties such as poros- ity. For permeabilities, simple averaging can lead to inaccurate and misleading results. In this paper we use the flow-based upscaling methods using local solutions of the equations $[17,19]$.

First, we briefly describe flow based upscaling methods. Consider the fine-scale permeability that is defined in the domain with underlying fine grid as shown in Figure 1. On the same graph we illustrate a coarsescale partition of the domain. To calculate the coarsescale permeability field at this level we need to determine it for each coarse block, $\Omega_{c}$. The coarse block permeability can be defined both using the solutions of local or global problems. The main idea used to calculate the coarse-scale permeability is that it should deliver the same average response as that of the underlying finescale problem, locally. The calculation of the coarsescale permeability based on local solutions is schematically depicted in Figure 1. For each coarse domain $\Omega_{c}$ we solve the local problems

$$
\nabla \cdot\left(\kappa_{f}(\mathbf{x}) \nabla \phi_{j}\right)=0,
$$

with some coarse-scale boundary conditions.

One of such boundary conditions is given by $\phi_{j}=1$ and $\phi_{j}=0$ on the opposite sides along the direction $e_{j}$ and no flow boundary conditions on all other sides, alternatively, $\phi_{j}=x_{j}$ on $\partial \Omega_{c}$. For these boundary conditions the coarse-scale permeability is given by

$$
\kappa_{c} \mathbf{e}_{j} \cdot \mathbf{e}_{l}=1 /\left|\Omega_{c}\right| \int_{\Omega_{c}} \kappa_{f}(\mathbf{x}) \nabla \phi_{j} \cdot \mathbf{e}_{l} d x,
$$

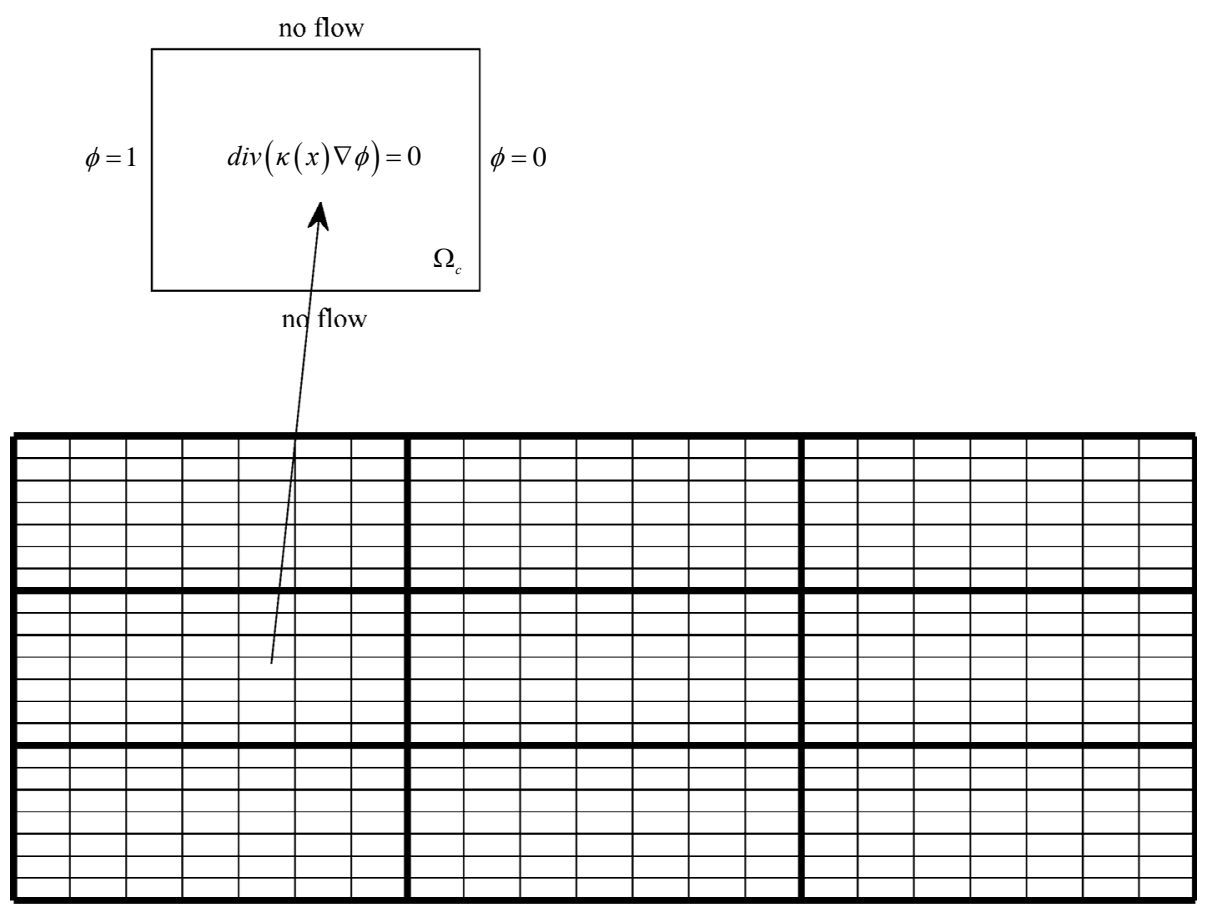

Figure 1. Schematic illustration of upscaling (not to scale): bold lines indicate a coarse-scale partitioning, while thin lines show a fine-scale partitioning within coarse-grid cells. In this paper, we upscaled a $50 \times 50$ fine-grid to a $5 \times 5$ coarse-grid. 
where $\phi_{j}$ is the solution of Equation (11) with prescribed boundary conditions. Various boundary condition can have some influence on the accuracy of the calculations, including periodic, Dirichlet, etc. These issues have been discussed for e.g., in [19]. In particular, for determining the coarse-scale permeability field one can choose local domains that are larger than target coarse block, $\Omega_{c}$, for Equation (11). Further Equation (12) is used in the domain $\Omega_{c}$, where $\phi_{j}$ are computed in the larger domains with correct scaling (see [19]). This way one reduces the effects of the artificial boundary conditions imposed on $\Omega_{c}$ (for details see [19]).

The use of the local solutions Equation (11) for determining the permeability field at different scales gives non-explicit relation for conditional distribution. We denote by $\mathcal{U}$ the local operator that maps the local fine-scale permeability field $\kappa_{f}$ into $\kappa_{c}$, defined as above. For our computations we assume

$$
\kappa_{c}=\mathcal{U}\left(\kappa_{f}\right)+\varepsilon
$$

where $\varepsilon$ are some random fluctuations that represent inaccuracies in the coarse-scale permeability. In reality, since we do not have the fine-scale field, $\kappa_{f}$ available, it is difficult to characterize the exact (nature of the) error in upscaling. However, one of the sources of these fluctuations are the errors associated with solving inverse problems on the coarse grid. The other source of the inaccuracies of measured coarse-scale permeability is due to the fact that the inversion on the coarse grid does not take into account the adequate form of the coarse-scale models. Here we assumed these errors to be normally distributed (further details follow in Section 4.2).

\section{Numerical Results}

For our numerical tests with the coarse-scale EnKF algorithm, we use a $50 \times 50$ fine grid (dimensionless domain size $50 \times 50$ ). We consider the coarse-scale permeability, which could be obtained by coarse-scale inversion of fractional flow data on a coarse grid [12,20]. This coarse-scale field could be thought as static data, which is to be honored as constraint (up to the data variance) in Equation (8), hence we need to always assimilate it in our coarse-scale EnKF algorithm.

An initial ensemble with different permeability realizations was generated using the sequential Gaussian simulation (SGSIM) ${ }^{1}$ [22]. We specified a Gaussian variogram model with a correlation length of 20 gridblocks in the $\mathrm{x}$-direction and 5 gridblocks in the y-direction; one of

${ }^{1}$ For reservoir simulation applications, the SGSIM has been used [21, 13] for generating initial ensemble members. This approach yields independent and identically distributed multivariate normal random fields (conditioned to well log data) the realizations is used as the reference field (depicted in Figure 2). The fractional flow will be calculated based on the fine-scale model in Section 2.1. Porosity $(\phi)$ is assumed to be equal to 0.15 for all grid blocks. For simplicity, relative permeabilities, $k_{r j}$ are assumed to be linear functions of water saturation $(S): k_{r w}(S)=S$, $k_{r o}(S)=1-S$. One injection well at the center of the field (injection rate: $71.4 \mathrm{~m}^{3} /$ day) and four producing wells at the four corners (all with equal rate of 17.85 $\mathrm{m}^{3}$ /day) were considered. The model equations are solved with no flow boundary conditions, zero initial water saturation, and discretizing the transport equation using first order upwind finite volume method. In Figure 3, we provide the predicted fractional flow for 256 initial ensemble members along with the true fractional flow (obtained from true permeability field).

To compare our proposed coarse-scale constrained EnKF results with the regular EnKF we will use the following mean $L_{2}$-norm error. Since we know the true (fine and coarse-scale) field for our synthetic problem, i.e., the true permeability field, denoting it by $\kappa^{\text {true }}$, the error for any ensemble member is

$$
\mathbf{e}^{(i)}=\kappa^{(i)}-\kappa^{\text {true }}, i=1,2, \cdots, N_{e} .
$$

Consider the $L_{2}$ norm of the error for each member, $\left\|\mathbf{e}^{(i)}\right\|_{2}=\sqrt{\sum_{j}\left[\mathbf{e}_{j}^{(i)}\right]^{2}}$, using which we define the mean $L_{2}$ error as

$$
\overline{\mathbf{e}}=\frac{1}{N_{e}} \sum_{i=1}^{N e}\left\|\mathbf{e}^{(i)}\right\|_{2},
$$

so that $\overline{\mathbf{e}}$ gives us an indication of the distance of entire ensemble from the true solution $\kappa^{\text {true }}$. Since after every

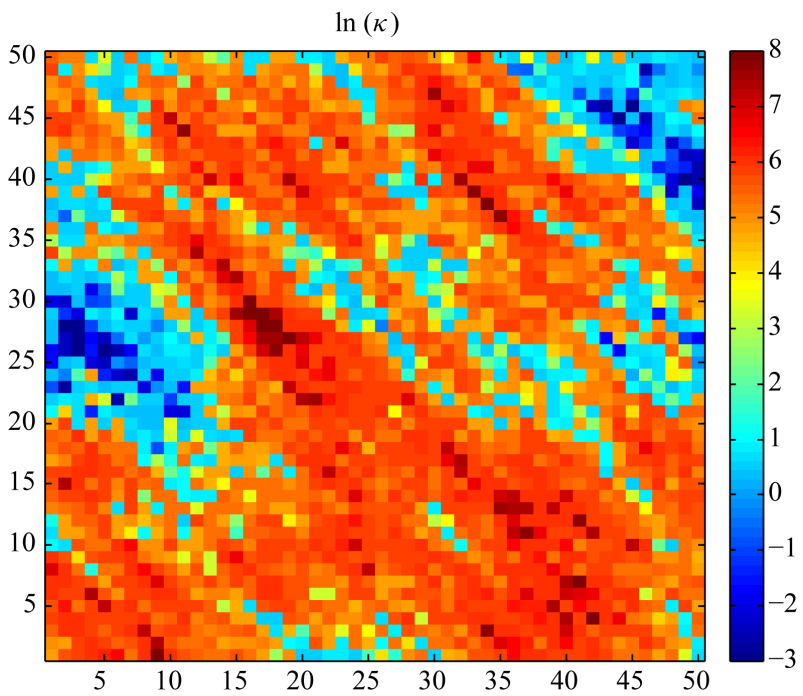

Figure 2. Natural logarithm of $50 \times 50$ true permeability field. 

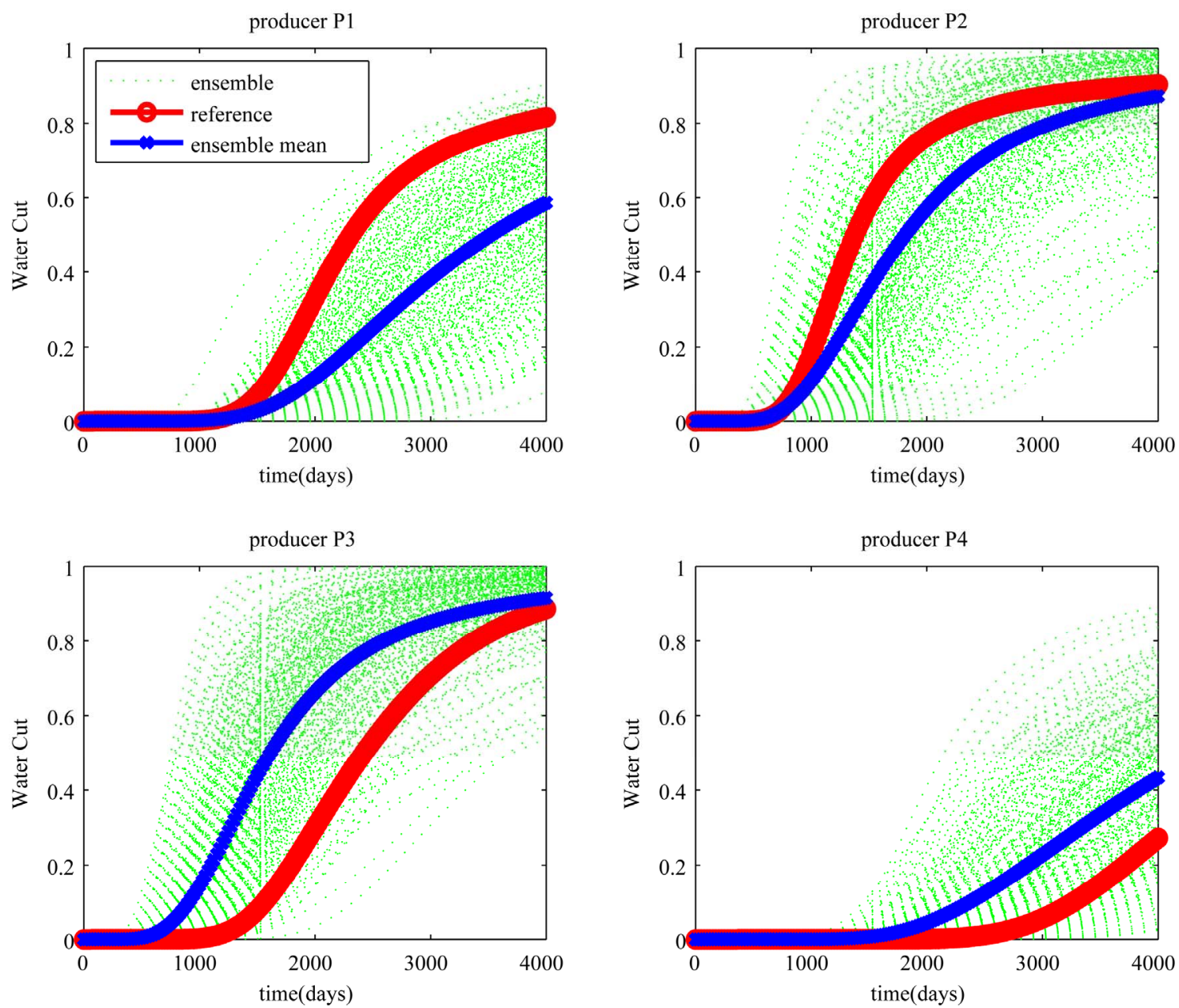

Figure 3. Fractional flow prediction with 256 initial ensemble members (no data assimilation); ensemble members (green dots), ensemble mean (blue crosses) compared with true water cut data (red open circles).

observation, we have updated ensemble members, therefore we can monitor the variation of $\overline{\mathbf{e}}$ over the time of assimilation; the success of assimilation can therefore be related to the decrease in $\overline{\mathbf{e}}$.

\subsection{EnKF with Fractional Flow Data Only}

We start with a presentation of results with regular EnKF, assimilating only water cut data. Next we will discuss results with the coarse-scale EnKF.

The water cut data from the reference field is assumed to be available every 200 days, with mean zero and standard deviation of 0.01 (therefore $\mathbf{R}^{1 / 2}=0.01 \mathbf{I}_{4}$, where $\mathbf{I}_{4}$ is unit matrix of size $4 \times 4$, since there are four producing wells). The observed data is assumed to be available up to 2400 days, hence we will perform assimilation between 200 and 2400 days. A prediction beyond interval of data assimilation, up to 4000 days is also provided. We selected an ensemble of size 256 for presenting our data assimilation results.
We assimilated the above described measured data, and using the assimilated permeability field, in Figure 4 we plot the assimilated water cut data along with the true data. Comparing with the initial forecast in Figure 3, we observe that the assimilated ensemble better envelopes the true data. We compare the initial permeability field before assimilation (Figure 5(a)-(d)) for a few ensemble members with the true field in Figure 2 and with those obtained after assimilation in Figure 6(a)-(d); note that the central, South East-North West channel is prominent but the features at the South West and North East corners are not well captured. Therefore assimilation of only water cut data helps in identifying only some of the important features.

\subsection{Coarse-Scale Constrained EnKF with Fractional Flow and Coarse-Scale Permeability Data}

In addition to water cut production data, the coarse-scale 

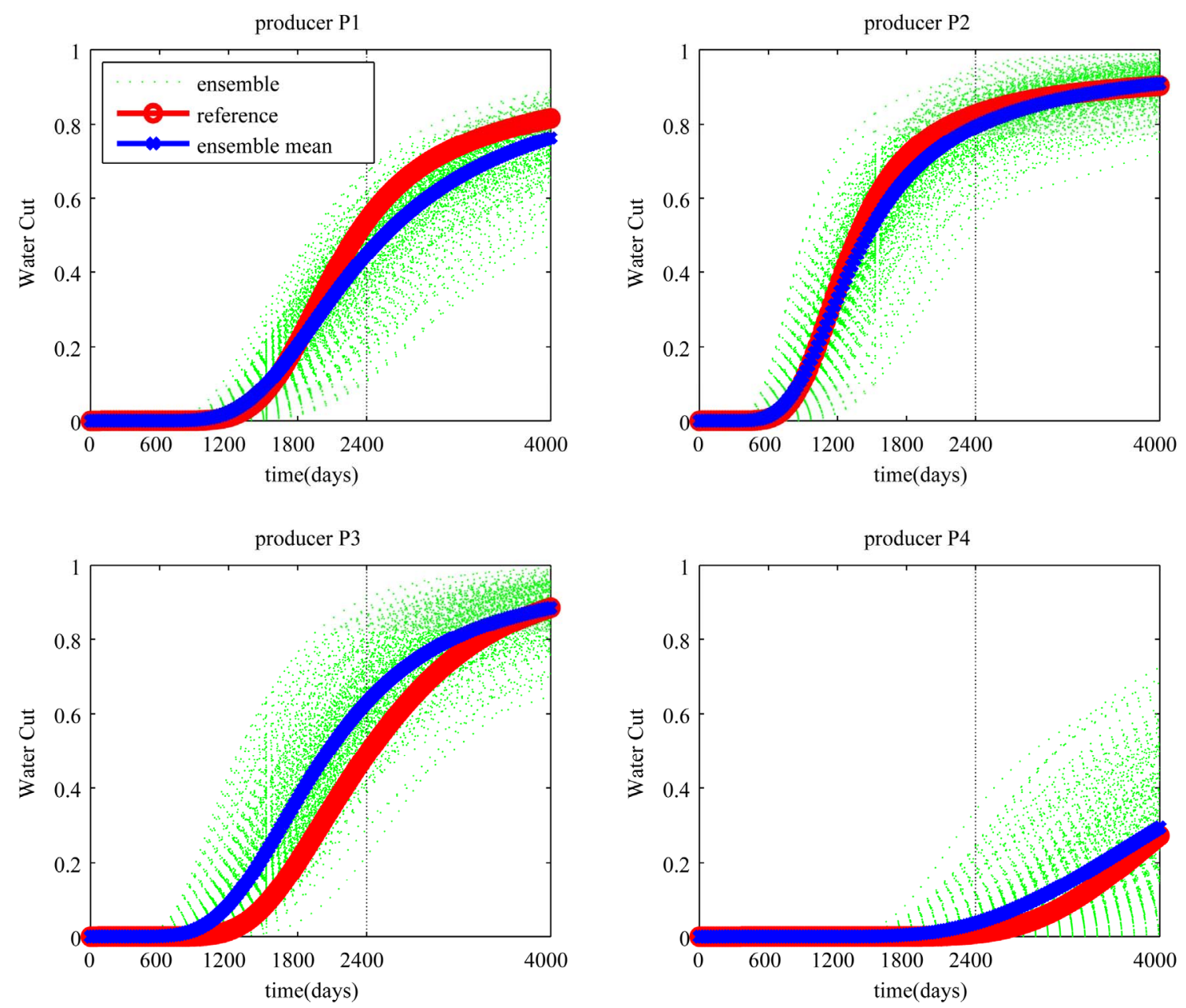

Figure 4. Water cut prediction using assimilated (regular EnKF for 2400 days) ensemble members, note the improved fit of ensemble when compared to that in Figure 3.

permeability data, as described in Section 3.1 has been used as additional measured data. Flow-based upscaling of reference permeability field is used as a proxy for inverted coarse field. Following our previous notation, this coarse-scale permeability data will be denoted by $\mathbf{z}$ (Equation (8)). The mapping between state variables (at fine-scale) and observations (at coarse-scale) is given by $\mathbf{U}=[\mathcal{U} \mathbf{0 0 0} 0], \mathcal{U}$, denotes flow-based upscaling.

Exactly as in the previous section, we prescribed the same frequency (of availability) and precision, $\mathbf{R}$ for the fractional flow data. Since we use coarse-scale permeability as additional data, it is to be assimilated whenever we assimilate water cut data. A $5 \times 5$ coarse-scale data with mean zero and variance, $Q=q I_{25}$ (we will present results with $q=4,2,1,0.5$ and 0.1 ,) so that we can consider the impact of coarse-scale data precision. In Figure 7 we plot the variation of mean $L_{2}$ error, $\overline{\mathbf{e}}$ (Equation (14)) with observation time, at the coarse-scale for different values of $q$. Figures 8(a) and (b) depict the correlation between coarse-scale ensemble mean and true fields for $q=4$ and 0.1 , respectively. As the precision of coarse-scale data is increased, i.e., for smaller variance, we observe a larger decrease in coarse-scale mean $L_{2}$ error and higher correlation with true coarse-scale field (correlation coefficient for $q=4,2,1,0.1$ respectively are $0.976,0.992,0.995,0.999$ ), because smaller variance Q implies more stricter coarse-scale data constraint in Equation (8). Figure 9(a)-(d) depict the fractional flow using the final permeability field after assimilation, for different coarse-scale data precisions. Figures 7 and 8(a)-(b) show that the coarse-scale data is being more accurately assimilated as it is made more precise. Also, notice the improved fit of ensemble prediction to the true data, for more precise coarse-scale data; also when compared to the regular EnKF results in Figure 4.

Now we discuss the results regarding fine-scale field. In Figure 10 we plot the fine-scale mean $L_{2}$ error for different values of $q$; the coarse-scale EnKF yields much lesser error than regular EnKF which assimilated only fractional flow data. The correlation coefficient 


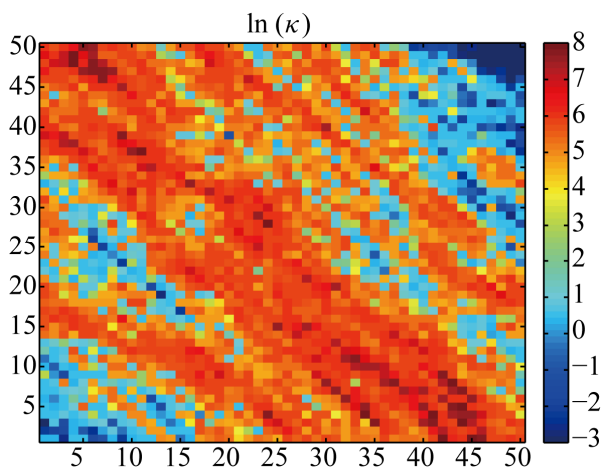

(a)

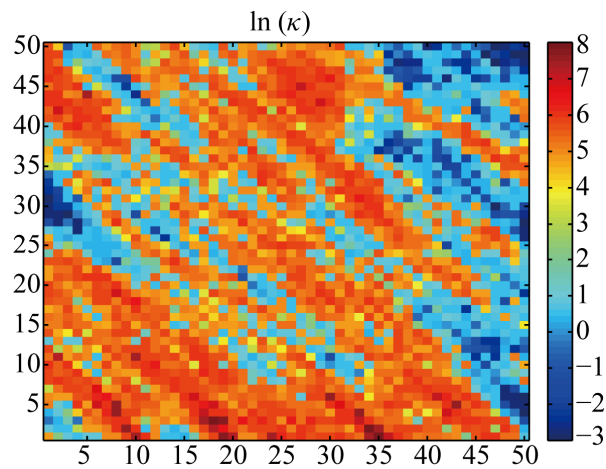

(c)

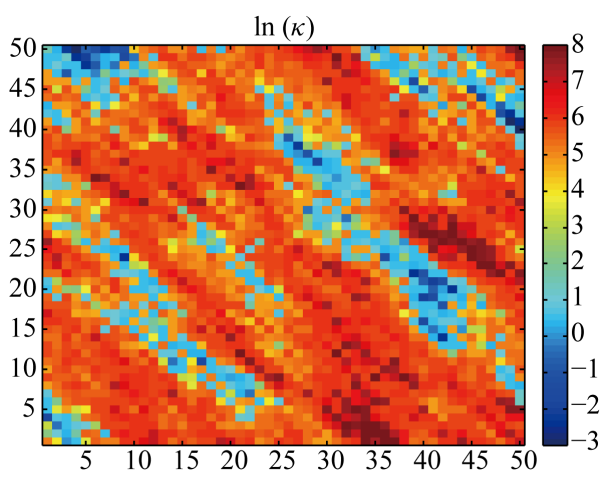

(b)

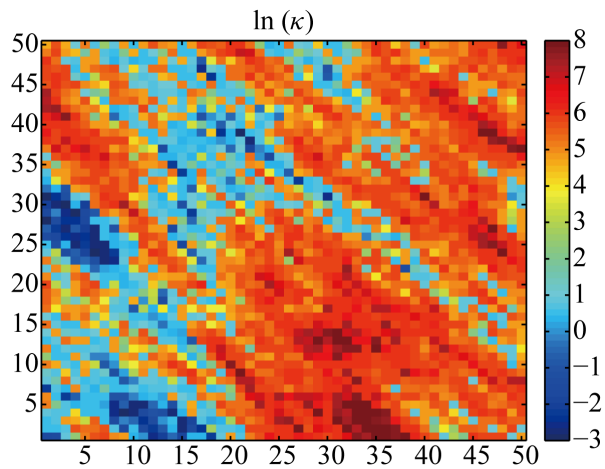

(d)

Figure 5. Log permeabilities of a few $i$-th. initial ensemble members (before data assimilation); left-right, (a) $i=50$, (b) 100, (c) 150, (d) 200.

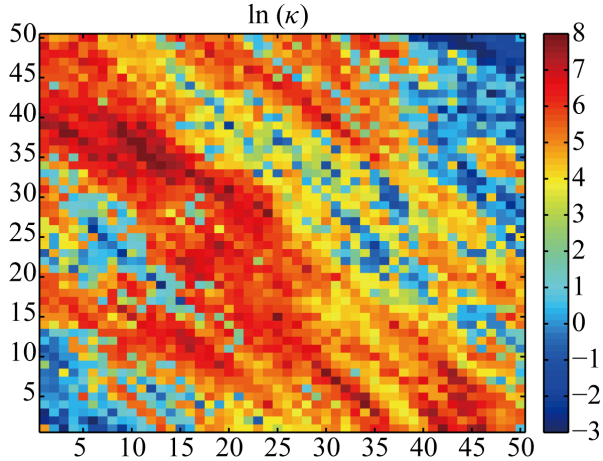

(a)

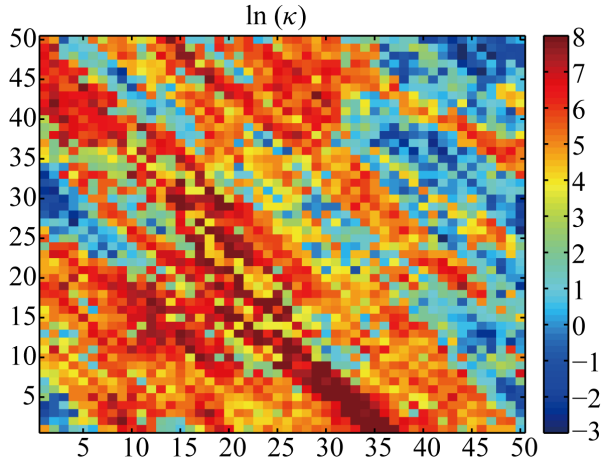

(c)

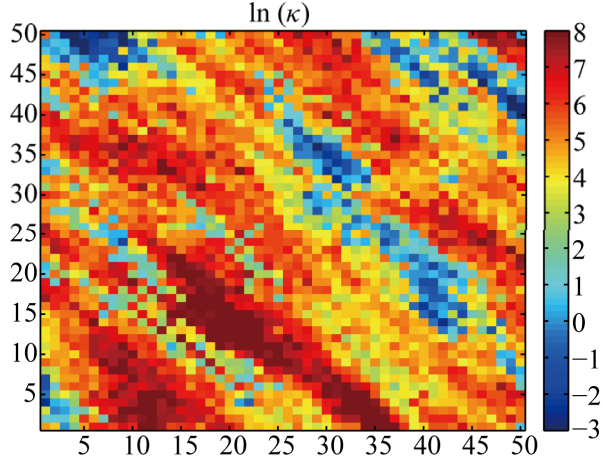

(b)

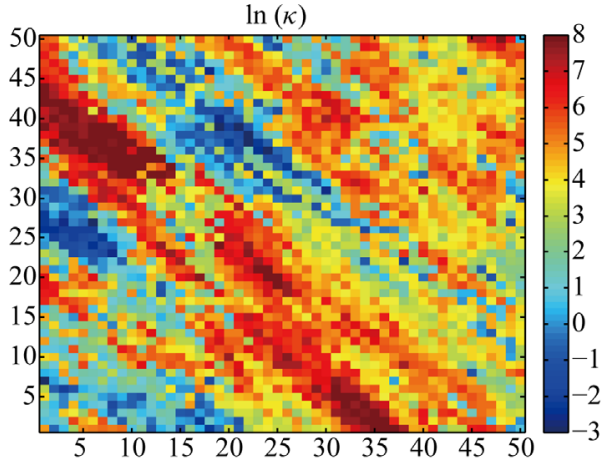

(d)

Figure 6. Same as above, but after assimilating water cut data with regular EnKF. 
Variation of mean $\mathrm{L}_{2}$ error at coarse-scale with observation time

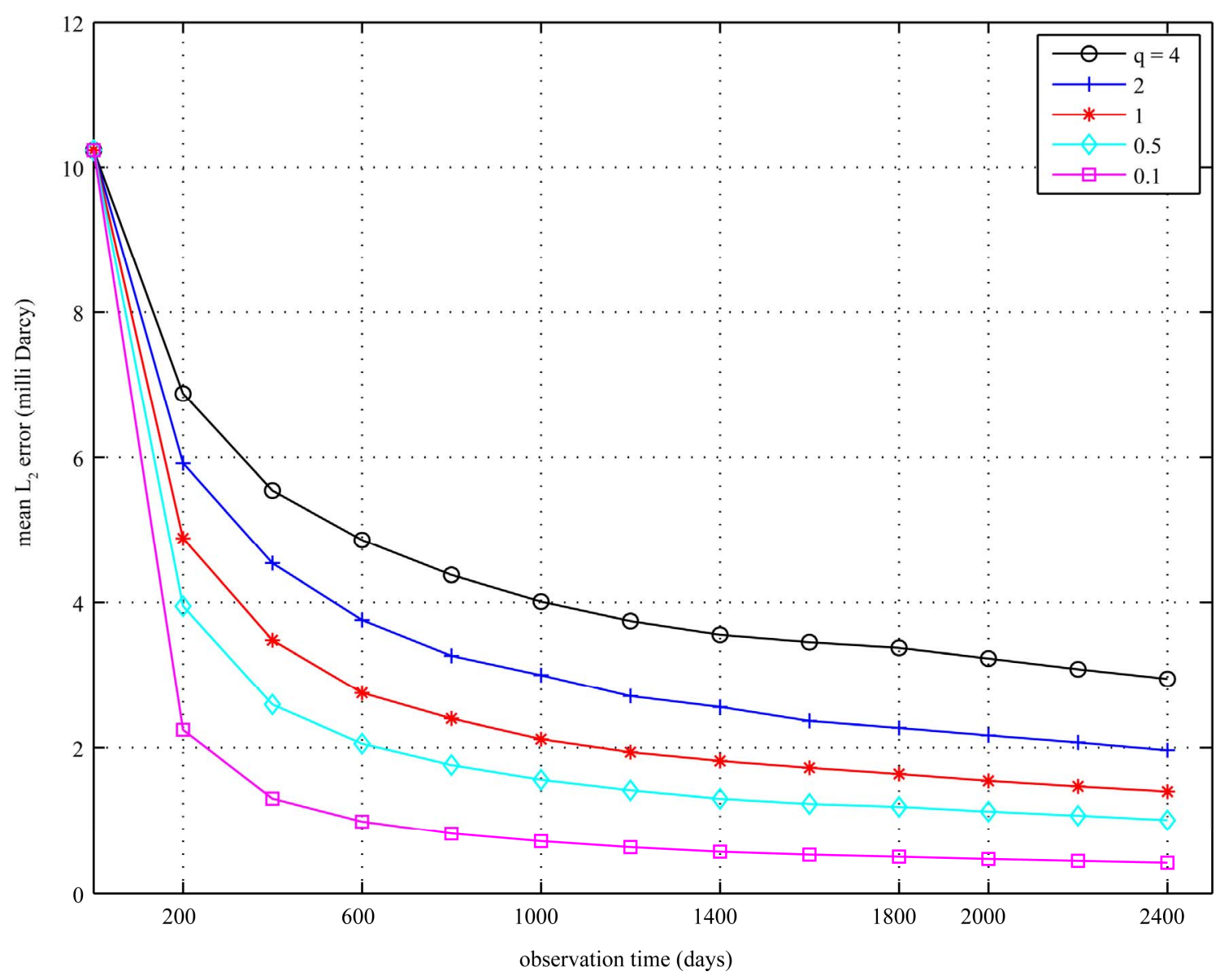

Figure 7. Decrease in $\overline{\mathrm{e}}$ computed at coarse-scale, as data (fractional flow and $5 \times 5$ coarse-scale permeability data at variance, $Q=q I)$ is assimilated using the coarse-scale EnKF algorithm.
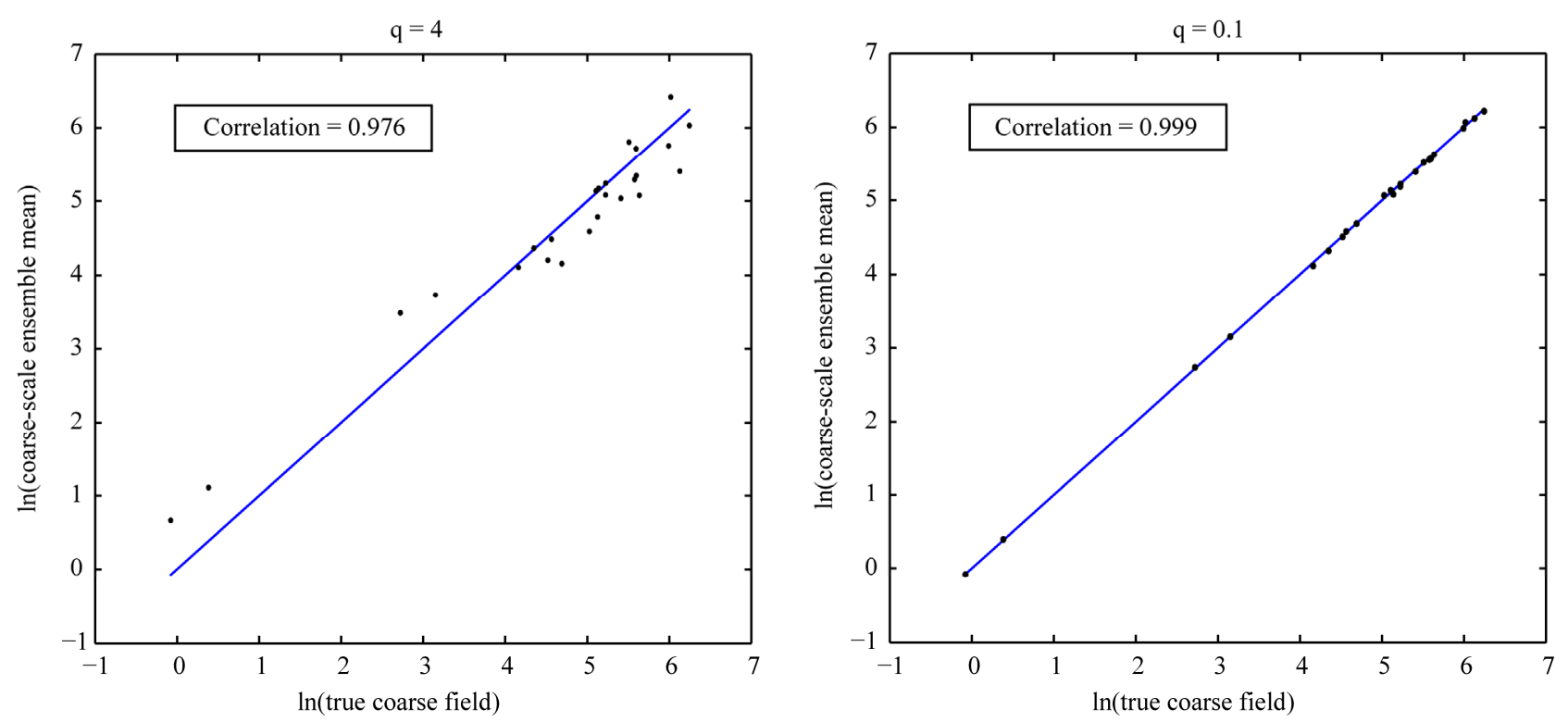

Figure 8. Correlation between coarse-scale ensemble mean and true permeability after assimilation for low and high precision in coarse data; (a) and (b): $q=4,0.1$. 

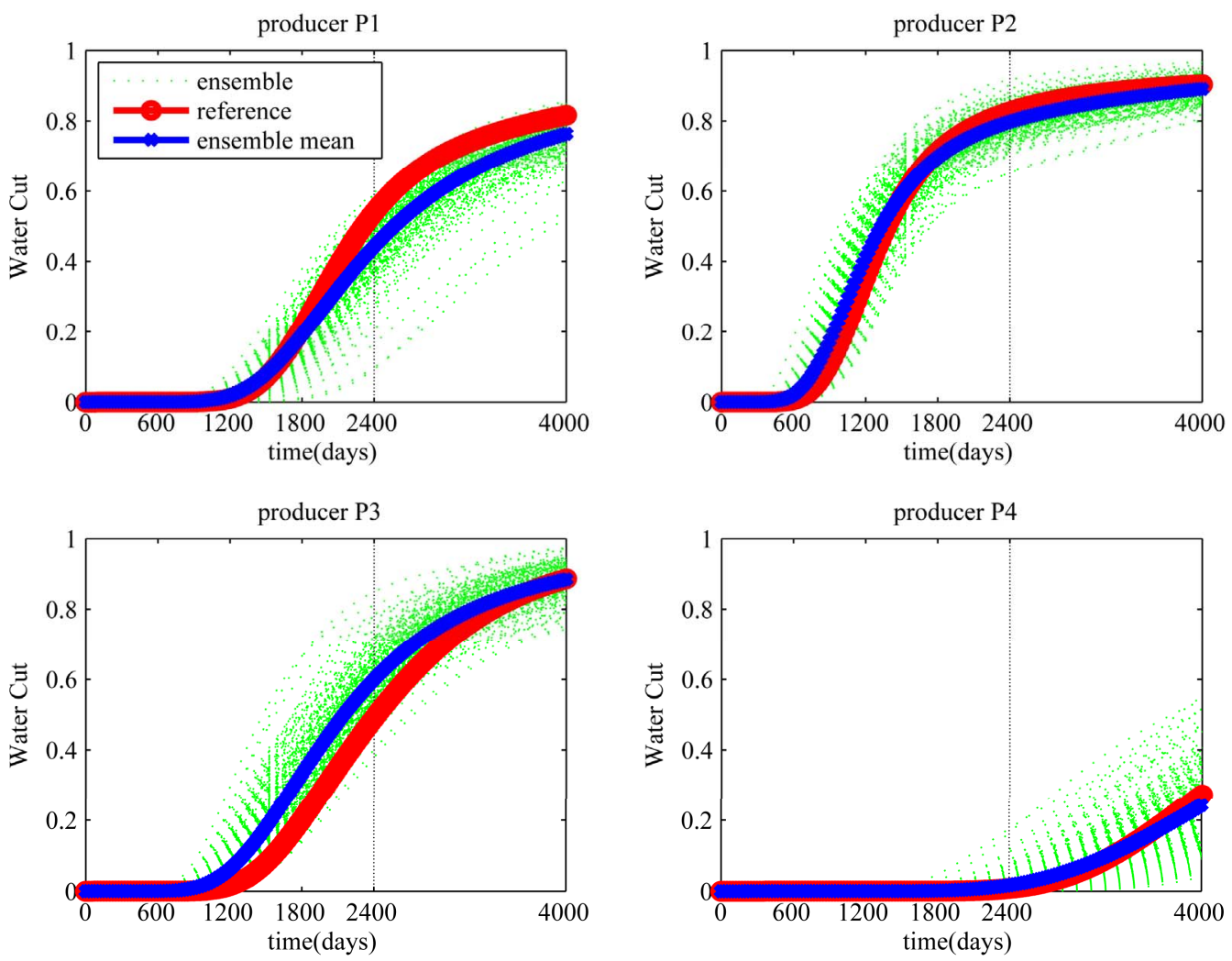

(a)
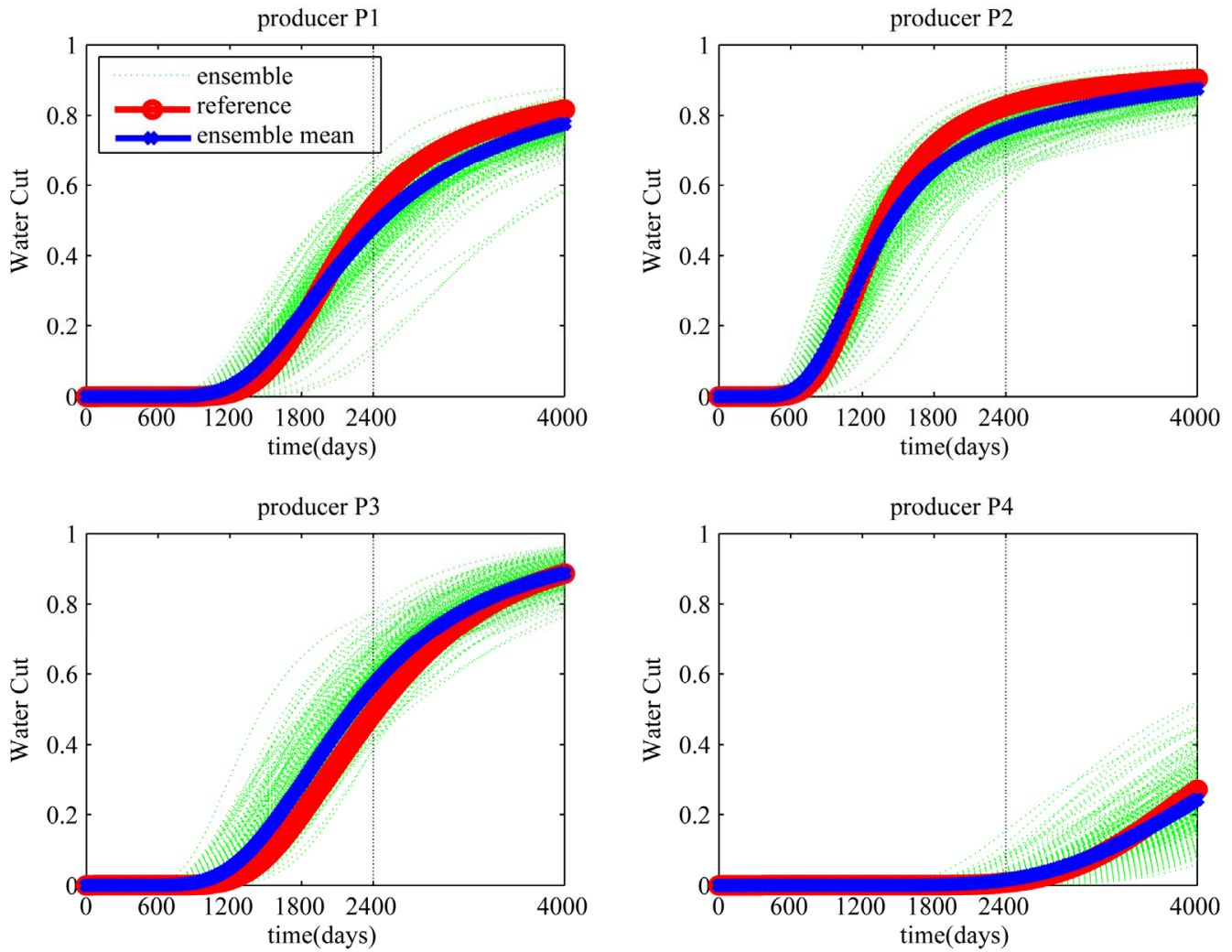

(b) 

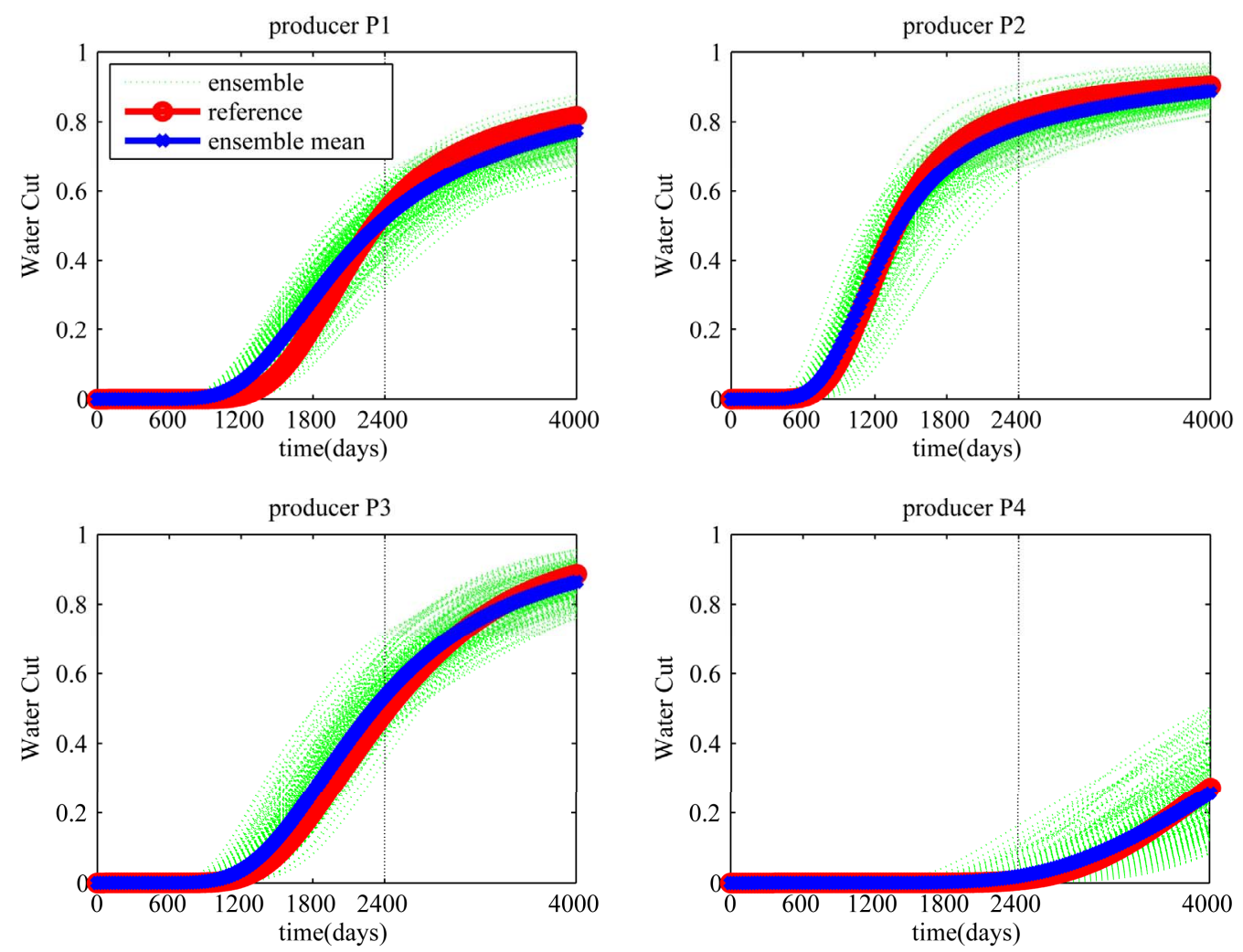

(c)
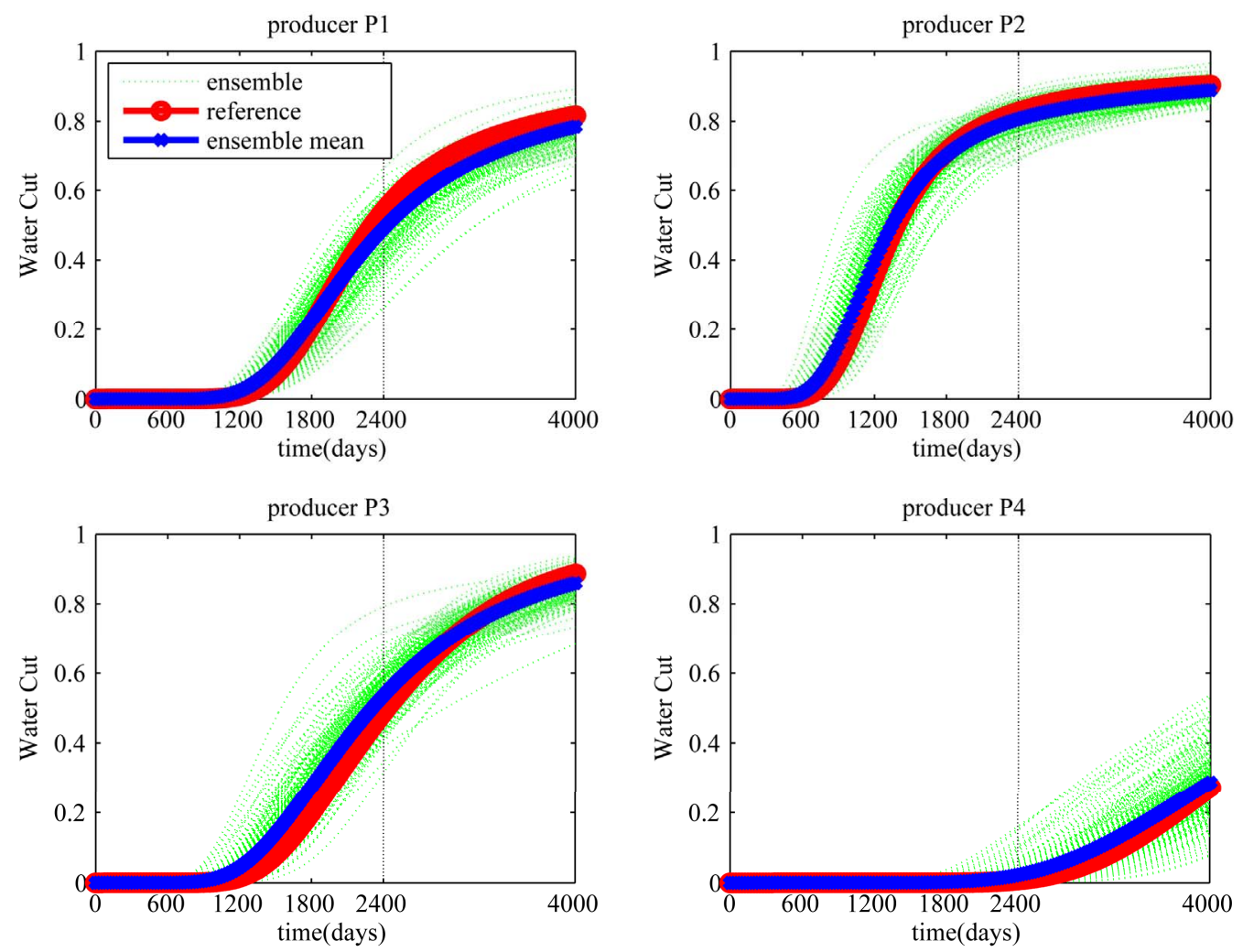

(d)

Figure 9. Same as in Figure 4, but using coarse-scale EnKF for data assimilation; clockwise, (a)-(d): $q=4,2,1,0.1$. 


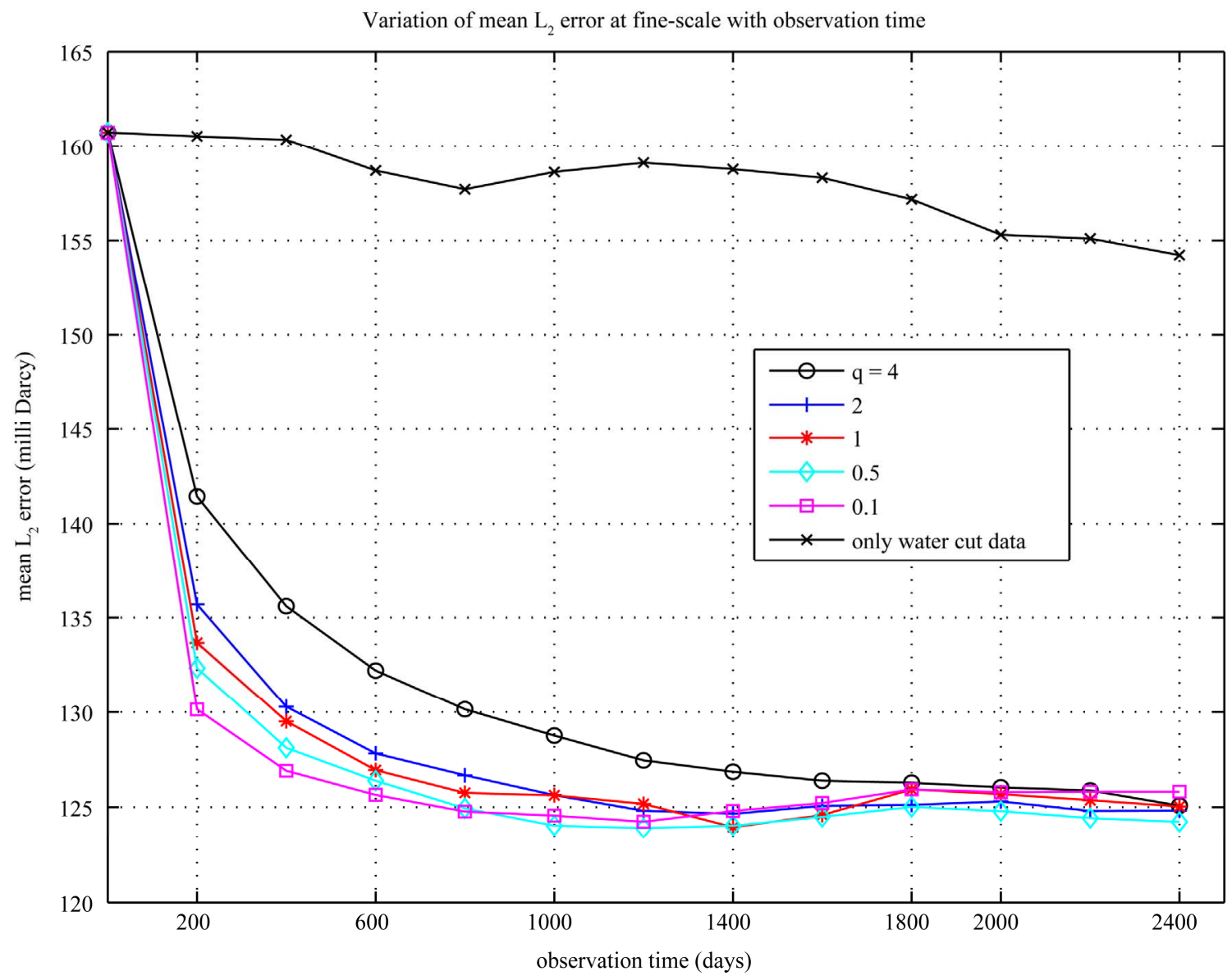

Figure 10. Same as in Figure 7, but at fine-scale, also shown is the error obtained with assimilation of fractional flow data only.

between fine-scale ensemble mean and true fields, after assimilating using regular EnKF is equal to 0.409, while with the coarse-scale EnKF for $q=4,2,1,0.1$, in that order were $0.644,0.652,0.638$ and 0.626 ; note higher correlation with the coarse-scale EnKF. We observe that higher precision, i.e., lower $q$ does not necessarily imply least $\overline{\mathbf{e}}$ or highest correlation, since highly precise coarse-scale data is relatively more weighted than the fractional flow data. Optimal value for the coarse-scale data variance can be obtained by prior calculation, which will be addressed in a future study.

The final permeability field, for a few ensemble members after assimilating with coarse-scale EnKF, for $q=1$ is shown in Figure 11(a)-(d); all shown samples seem to be more closer to the true field (Figure 2) than those obtained with regular EnKF (Figure 6(a)-(d)). In particular note that the low permeability region at the North East and high permeability at the South West corners are well captured.

\section{Conclusions}

The EnKF is increasingly being used for subsurface cha- racterization in various geological and groundwater applications to identify fine-scale state and parameters. So far, various implementations have been based on using dynamic, production data, such as water cut, well pressures, etc, for sequential data assimilation. Only recently dynamic data other than production data has been considered in the EnKF context ([23,24]), nevertheless the observed data to be assimilated was assumed to be at the finest scale. For a number of reasons, it is widely recognized that usage of additional multiscale data could further reduce the uncertainty at the fine scale. This is further motivated by the increasing popularity of coarsescale modeling. In this light, here we proposed assimilation of coarse-scale data along with water cut, production data using coarse-scale EnKF. The modification to the regular EnKF (assimilation of only water cut data) is completely recursive and easily implementable. The relation between fine and coarse scales has been modeled via physics based upscaling, which could be thought of as a nonlinear observation operator linking the coarsescale data to the unknown fine-scale variables. In addition, the proposed methodology could be used in any other sequential data assimilation method as well and also 


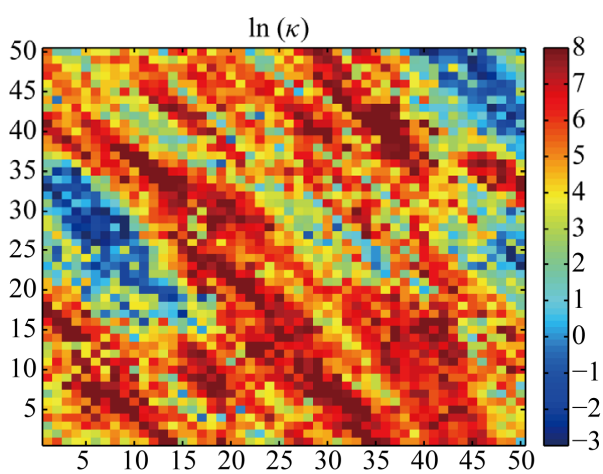

(a)

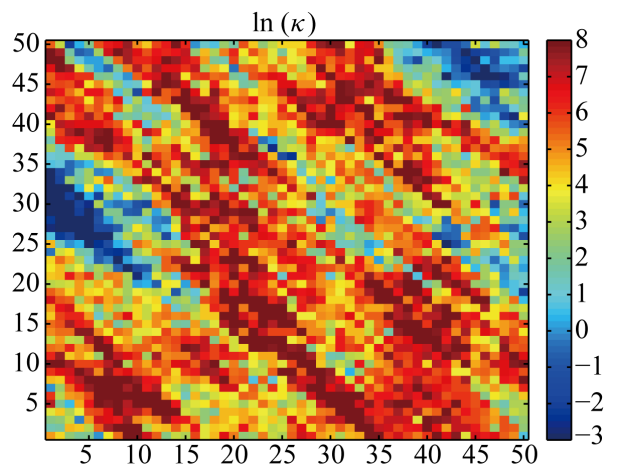

(c)

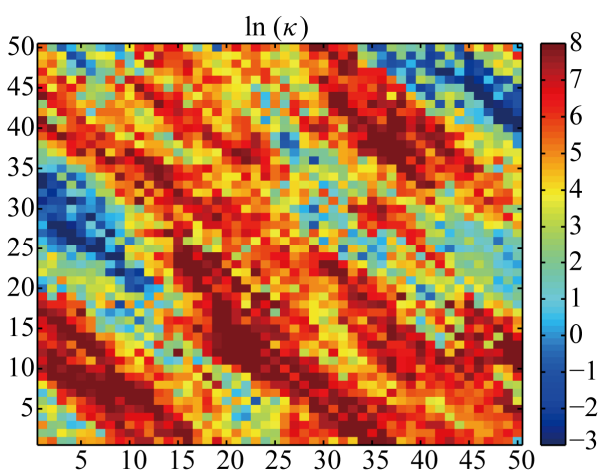

(b)

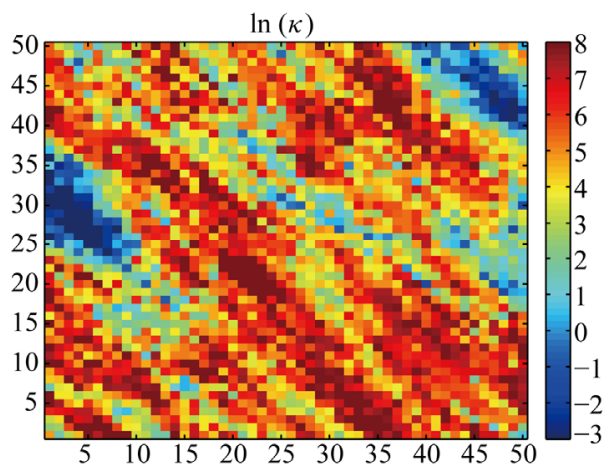

(d)

Figure 11. Same as in Figure 6, but assimilated using coarse-scale EnKF with $q=1$ for the variance of coarse-scale permeability data.

also with any other upscaling method.

The coarse-scale EnKF was tested and compared with the regular EnKF for a 2D synthetic $50 \times 50$ heterogenuous true field. We considered coarse-scale permeability data as additional data on a $5 \times 5$ coarse grid. This coarse-scale data was always assimilated along with water cut data. The data variance was varied from low to high, to study its impact on assimilated results. In all cases, we observed that the assimilated, ensemble mean coarse-scale field for all variances was highly correlated to the true coarse-scale field. In addition, lower variance in the coarse-scale data yielded higher correlation. The water cut data was better honored, both for higher precision of coarse data, and when compared with regular EnKF. As for the fine-scale permeability field, the coarse-scale EnKF yielded lesser error in an averaged $L_{2}$ norm, error taken w.r.t. the reference field. In addition, a few individual samples were picked to compare the assimilated fields with different EnKF procedures; experiment with coarse-scale permeability data provided final samples which captured most closely the features in the reference fine-scale field.

Though in our current paper we used only one coarsescale, the proposed method can be easily implemented to integrate as many scales as required by the available data and is independent of the underlying fine-scale field. Based on our results we conclude that there could be a singinificant improvement in subsurface characterization if accurate and additional data at coarse-scales is available. In future we plan to study the nature of errors involved in coarse-scale data and upscaling and in-turn their influence on the proposed coarse-scale EnKF.

\section{References}

[1] J. M. Lewis, S. Lakshmivarahan and S. Dhall, "Dynamic Data Assimilation: A Least Squares Approach (Encyclopedia of Mathematics and its Applications)," Cambridge University Press, Cambridge, 2006. doi:10.1017/CBO 9780511526480

[2] G. Evensen, "Data Assimilation: The Ensemble Kalman Filter,” Springer, Berlin, 2006.

[3] Y. Liu and H. V. Gupta, "Uncertainty in Hydrologic Modeling: Toward and Integrated Data Assimilation Framework," Water Resources Research, Vol. 43, 2007, W07401. doi:10.1029/2006WR005756

[4] D. E. Lumley, "Time-Lapse Seismic Reservoir Monitoring," Geophysics, Vol. 66, No. 1, 2001, pp. 50-53. doi:10.1190/1.1444921

[5] G. Nævdal, L. Johnson, S. Aanonsen and E. Vefring, "Reservoir Monitoring and Continuous Model Updating 
Using Ensemble Kalman Filter,” SPE Journal, Vol. 10, No. 1, 2005, pp. 66-74.

[6] X.-H Wen and W. H. Chen, "Real-Time Reservoir Model Updating Using Ensemble Kalman Filter,” Proceedings of SPE Reservoir Simulation Symposium 2005, SPE 92991, 31 January-2 February 2005, Texas.

[7] Y. Q. Gu and D. S. Oliver, "The Ensemble Kalman Filter for Continuous Updating of Reservoir Simulation Models,” Journal of Energy Resources Technology-Transactions of the ASME, Vol. 128, 2006, pp. 79-87. doi:10.1115/1.2134735

[8] S. H. Lee, A. Malallah, A. Datta-Gupta and D. Higdon, "Multiscale Data Integration Using Markov Random Fields," SPE Reservoir Evaluation Engineering, Vol. 5, 2002, pp. 68-78. doi:10.2118/76905-PA

[9] Y. Efendiev, A. Datta-Gupta, V. Ginting, X. Ma and B. Mallick, "An Efficient Two-Stage Markov Chain Monte Carlo Method for Dynamic Data Integration,” Water Resources Research, Vol. 41, 2005, doi:10.1029/2004WR003764

[10] M. Christie, “Upscaling for Reservoir Simulation,” Journal of Petroleum Technology, Vol. 48, No. 11, 1996, pp. 1004-1010. doi:10.2118/37324-MS

[11] J. W. Barker and S. Thibeau, "A Critical Review of the Use of Pseudo-Relative Permeabilities for Upscaling," SPE Reservoir Engineering, Vol. 12, 1997, pp.138-143. doi:10.2118/35491-PA

[12] Y. Efendiev, A. Datta-Gupta, I. Osako and B. Mallick, "Multiscale Data Integration Using Coarse-Scale Models," Advances in Water Resources, Vol. 28, No. 3, 2005, pp. 303-314. doi:10.1016/j.advwatres.2004.10.013

[13] D. Devegowda, E. Arroyo-Negrete and A. Datta-Gupta, "Efficient and Robust Reservoir Model Updating Using Ensemble Kalman Filter with Sensitivity-Based Covariance Localization," Proceedings of SPE Reservoir Simulation Symposium, Houston, February 2007. doi:10.2118/106144-MS

[14] G. Evensen, "Sequential Data Assimilation with a Nonlinear Quasi-Geostrophic Model Using Monte Carlo Methods to Forecast Error Statistics," Journal of Geophysical Research, Vol. 99, 1994, pp. 143-162. doi:10.1029/94JC00572

[15] G. Burgers, P. J. van Leeuwen and G. Evensen, “Analysis
Scheme in the Ensemble Kalman Filter," Monthly Weather Review, Vol. 126, No. 6, 1998, pp. 1719-1724. doi:10.1175/1520-0493(1998)126<1719:ASITEK>2.0.C $\mathrm{O} ; 2$

[16] Y. Chen and D. Zhang, "Data Assimilation for Transient Flow in Geologic Formations Via Ensemble Kalman Filter," Advances in Water Resources, Vol. 29, 2006, pp. 1107-1122. doi:10.1016/j.advwatres.2005.09.007

[17] L. J. Durlofsky, "Numerical Calculation of Equivalent Grid Block Permeability Tensors for Heterogeneous Porous Media,” Water Resources Research, Vol. 27, No. 5, 1991, pp. 699-708. doi:10.1029/91WR00107

[18] L. J. Durlofsky, "Coarse Scale Models of Two Phase Flow in Heterogeneous Reservoirs: Volume Averaged Equations and Their Relationship to the Existing Upscaling Techniques,” Computational Geosciences, Vol. 2, 1998, pp. 73-92. doi:10.1023/A:1011593901771

[19] X. H. Wu, Y. R. Efendiev and T. Y. Hou, "Analysis of Upscaling Absolute Permeability," Discrete and Continuous Dynamical Systems, Series B, Vol. 2, 2002, pp. 185-204. doi:10.3934/dcdsb.2002.2.185

[20] S. Yoon, A. H. Malallah, A. Datta-Gupta, D. W. Vasco and R. A. Behrens, "A Multiscale Approach to Production-Data Integration Using Streamline Models,” SPE Journal, Vol. 6, No. 2, 2001, pp. 182-192.

[21] Y. Gu and D. S. Oliver, "History Matching of the PUNQ-S3 Reservoir Model Using the Ensemble Kalman Filter,” SPE Journal, Vol. 10, No. 2, 2005, pp. 217-224. doi:10.2118/89942-PA

[22] C. V. Deutsch, "Journel A. GSLIB: Geostatistical Software Library and User's Guide,” Oxford Univiversity Press, Oxford, 1992.

[23] Y. Dong , Y. Gu and D. S. Oliver, "Sequential Assimilation of 4D Seismic Data for Reservoir Description Using the Ensemble Kalman Filter," Journal of Petroleum Science and Engineering, Vol. 53, 2006, pp. 83-99. doi:10.1016/j.petrol.2006.03.028

[24] J.-A. Skjervheim, G. Evensen, S. Aanonsen, B. Ruud and T. Johansen, "Incorporating 4D Seismic Data in Reservoir Simulation Models Using Ensemble Kalman Filter," SPE Journal, Vol. 12, No. 3, 2007, pp. 282-292. doi:10.2118/95789-PA 


\section{Appendix A: Two Step Coarse-Scale Constrained Kalman Filter Estimate}

From Section 3,

$$
\mathcal{J}_{f}=\frac{1}{2}(\boldsymbol{\Psi}-\overline{\boldsymbol{\Psi}})^{T}\left(\mathbf{P}^{f}\right)^{-1}(\boldsymbol{\Psi}-\overline{\boldsymbol{\Psi}})
$$

and

$$
\mathcal{J}_{y}=\frac{1}{2}(\mathbf{y}-\mathbf{H}[\boldsymbol{\Psi}])^{T} \mathbf{R}^{-1}(\mathbf{y}-\mathbf{H}[\boldsymbol{\Psi}])
$$

For notational simplicity we will denote $\boldsymbol{\mu}_{\boldsymbol{\Psi}}$ as $\boldsymbol{\mu}$ and denote $\mathbf{P}^{f}$ by $\mathbf{B}$.

Step 1 (minimize $\mathcal{J}_{f}+\mathcal{J}_{y}$ ):

First we minimize the sum, $\mathcal{J}_{1}=\mathcal{J}_{f}+\mathcal{J}_{y}$. The gradient $^{2}$ of above quadratic cost functional with respect to (w.r.t.) $\boldsymbol{\Psi}$ is given by

$$
\nabla_{\boldsymbol{\Psi}} \mathcal{J}_{1}=\mathbf{B}^{-1}(\boldsymbol{\Psi}-\boldsymbol{\mu})-\mathbf{H}^{T} \mathbf{R}^{-1}(\mathbf{y}-\mathbf{H}[\boldsymbol{\Psi}])
$$

Then the minimizer $\tilde{\boldsymbol{\mu}}$, of $\mathcal{J}_{1}$ satisfies (we assume $\mathbf{H}$ to be linear)

$$
\mathbf{B}^{-1}(\tilde{\boldsymbol{\mu}}-\boldsymbol{\mu})-\mathbf{H}^{T} \mathbf{R}^{-1}(\mathbf{y}-\mathbf{H} \tilde{\boldsymbol{\mu}})=0 .
$$

Rearranging the above equation we get,

$$
\left[\mathbf{B}^{-1}+\mathbf{H}^{T} \mathbf{R}^{-1} \mathbf{H}\right] \tilde{\boldsymbol{\mu}}=\mathbf{B}^{-1} \boldsymbol{\mu}+\mathbf{H}^{T} \mathbf{R}^{-1} \mathbf{y}
$$

Note that the Hessian of $\mathcal{J}_{1}$ w.r.t. $\boldsymbol{\Psi}$ is given by $\mathbf{B}^{-1}+\mathbf{H}^{T} \mathbf{R}^{-1} \mathbf{H}$ and for linear quadratic cost functionals, the Hessian inverse is equal to the error covariance matrix. Therefore the error covariance matrix, $\tilde{\mathbf{B}}$ for $\tilde{\boldsymbol{\mu}}$ is given by

$$
\tilde{\mathbf{B}}=\left[\mathbf{B}^{-1}+\mathbf{H}^{T} \mathbf{R}^{-1} \mathbf{H}\right]^{-1}
$$

Step 2 (minimize $\mathcal{J}_{g}+\mathcal{J}_{z}$ ):

We use $\tilde{\boldsymbol{\mu}}, \tilde{\mathbf{B}}$ in

$$
\begin{gathered}
\mathcal{J}_{g}=\frac{1}{2}(\boldsymbol{\Psi}-\tilde{\boldsymbol{\mu}})^{T}(\tilde{\mathbf{B}})^{-1}(\boldsymbol{\Psi}-\tilde{\boldsymbol{\mu}}) . \\
\mathcal{J}_{z}=\frac{1}{2}(\mathbf{z}-\mathbf{U}[\boldsymbol{\Psi}])^{T} Q^{-1}(\mathbf{z}-\mathbf{U}[\boldsymbol{\Psi}]) .
\end{gathered}
$$

Therefore the minimum $\tilde{\boldsymbol{\mu}}$, of $\mathcal{J}_{g}+\mathcal{J}_{z}$ satisfies

$$
\left[(\tilde{\mathbf{B}})^{-1}+\mathbf{U}^{T} \mathbf{Q}^{-1} \mathbf{U}\right] \hat{\boldsymbol{\mu}}=(\tilde{\mathbf{B}})^{-1} \tilde{\boldsymbol{\mu}}+\mathbf{U}^{T} \mathbf{Q}^{-1} \mathbf{z} .
$$

Using Equations (16) and (15) we can rewrite above as

$$
\begin{aligned}
& \underbrace{\left[\mathbf{B}^{-1}+\mathbf{H}^{T} \mathbf{R}^{-1} \mathbf{H}\right.}_{(\tilde{\mathbf{B}})^{-1}}+\mathbf{U}^{T} \mathbf{Q}^{-1} \mathbf{U}] \hat{\boldsymbol{\mu}} \\
& =\underbrace{\mathbf{B}^{-1} \boldsymbol{\mu}+\mathbf{H}^{T} \mathbf{R}^{-1} \mathbf{y}}_{\text {r.h.s of weqation (14) }}+\mathbf{U}^{T} \mathbf{Q}^{-1} \mathbf{z} .
\end{aligned}
$$

${ }^{2}$ We note in passing that $\mathbf{B}$ and $\mathbf{R}$ are covariance matrices and are positive definite by construction, and hence for our derivation purposes, are formally invertible.
It is trivial to show that $\hat{\boldsymbol{\mu}}$ also satisfies

$$
\nabla_{\boldsymbol{\Psi}}\left[\mathcal{J}_{f}+\mathcal{J}_{y}+\mathcal{J}_{z}\right]=0 .
$$

Therefore the two step method to obtain the final estimate $\hat{\boldsymbol{\mu}}$, gives the same results as a one shot approach of minimizing $\mathcal{J}_{f}+\mathcal{J}_{y}+\mathcal{J}_{z}$.

\section{Appendix B: The Coarse-Scale EnKF Algorithm}

Run the simulation model up to a particular observation time for entire ensemble to get predicted samples:

$$
\left\{\boldsymbol{\Psi}^{(i)}\right\}_{i=1}^{N_{e}}, \quad \mathbf{A}=\left(\boldsymbol{\Psi}^{(1)}, \boldsymbol{\Psi}^{(2)}, \cdots, \boldsymbol{\Psi}^{\left(N_{e}\right)}\right) .
$$

1) Step 1: Using measured water cut data $\mathbf{y}$ with vari-

ance $\mathbf{R}$, get updated ensemble: $\left\{\widetilde{\boldsymbol{\Psi}}^{(i)}\right\}_{i=1}^{N_{e}}$,

- Step 1.1 Find ensemble mean (Equation (4)), $\overline{\boldsymbol{\Psi}}$.

- Step 1.2 Subtract deviation from mean $\mathbf{A}^{\prime}=\left(\mathbf{b}^{(1)}, \mathbf{b}^{(2)}, \cdots, \mathbf{b}^{\left(N_{e}\right)}\right), \quad \mathbf{b}^{(i)}=\boldsymbol{\Psi}^{(i)}-\overline{\boldsymbol{\Psi}}$.

- Step 1.3 Apply $\mathbf{H}$ to each column of $\mathbf{A}^{\prime}$ to get $\mathbf{S}=\mathbf{H} \mathbf{A}^{\prime}$. i.e., simply pick the water cut deviations in $\mathbf{A}^{\prime}$.

- Step 1.4 for $i=1,2, \cdots, N_{e}$, Sample $v^{(i)} \stackrel{\text { i.i.d. }}{\sim} \mathcal{N}(\mathbf{0}, \mathbf{R})$.

$$
\begin{aligned}
& \mathbf{y}^{(i)}=\mathbf{y}+v^{(i)}, \\
& \mathbf{R}^{1 / 2}=\left(v^{(1)}, v^{(2)}, \cdots, v^{\left(N_{e}\right)}\right), \\
& \mathbf{D}=\left(\mathbf{d}^{(1)}, \mathbf{d}^{(2)}, \cdots, \mathbf{d}^{\left(N_{e}\right)}\right), \quad \mathbf{d}^{(i)}=\mathbf{y}^{(i)}-\mathbf{W}_{c}^{(i)} ; \quad \mathbf{W}_{c}^{(i)}
\end{aligned}
$$

is predicted water cut for each ensemble member. end for

- Step 1.5 Compute SVD $\left[\mathbf{S}+\mathbf{R}^{1 / 2}\right]=\mathbf{X}_{L} \Sigma \mathbf{X}_{R}$.

Get $\hat{\Sigma}$ retaining first few singular values which explain most variability in $\Sigma$, corresponding left singular vectors: $\mathbf{X}_{L}$.

- $\quad$ Step 1.6 Update ensemble: Eqnuation (7),

$$
\begin{aligned}
\widetilde{\mathbf{A}} & =\left(\widetilde{\boldsymbol{\Psi}}^{(1)}, \widetilde{\boldsymbol{\Psi}}^{(2)}, \cdots, \widetilde{\boldsymbol{\Psi}}^{\left(N_{e}\right)}\right), \\
\widetilde{\mathbf{A}} & =\mathbf{A}+\mathbf{A}^{\prime} \mathbf{S}^{T} \hat{\mathbf{X}}_{L} \hat{\Sigma}-{ }^{2} \hat{\mathbf{X}}_{L}^{T} \mathbf{D} .
\end{aligned}
$$

2) Step 2: Using coarse-scale data $\mathbf{z}$ with variance $\mathbf{Q}$, get updated ensemble: $\left\{\widehat{\boldsymbol{\Psi}}^{(i)}\right\}_{i=1}^{N_{e}}$.

- Step 2.1 Compute coarse-scale ensemble prediction: $\mathbf{u}^{(i)}=\mathbf{U} \widetilde{\Psi}^{(i)}, i=1,2, \cdots, N_{e}$.

- Step 2.2 Coarse-scale mean: $\boldsymbol{\mu}^{\prime}=\frac{1}{N_{e}} \sum_{i=1}^{N_{e}} \mathbf{u}^{(i)}$.

- $\quad$ Step 2.3 Coarse-scale deviations: $\mathbf{S}^{\prime}=\left(\mathbf{s}^{(1)}, \mathbf{s}^{(2)}, \cdots, \mathbf{s}^{\left(N_{e}\right)}\right), \quad \mathbf{s}^{(i)}=\mathbf{u}^{(i)}-\boldsymbol{\mu}^{\prime}$

- Step 2.4 Repeat Step 1.4, using coarse-scale mea- 
surement. for $i=1,2, \cdots, N_{e}$,

Sample $\omega^{(i)} \stackrel{\text { i.i.d. }}{\sim} \mathcal{N}(\mathbf{0}, \mathbf{R})$.

$\mathbf{z}^{(i)}=\mathbf{z}+\omega^{(i)}$,

$\mathbf{Q}^{1 / 2}=\left(\omega^{(1)}, \omega^{(2)}, \cdots, \omega^{\left(N_{e}\right)}\right)$,

$\mathbf{D}^{\prime}=\left(\mathbf{d}^{(1)}, \mathbf{d}^{(2)}, \cdots, \mathbf{d}^{\left(N_{e}\right)}\right), \quad \mathbf{d}^{(i)}=\mathbf{z}^{(i)}-\mathbf{u}^{(i)}$. end for

- Step 2.5 Compute SVD $\left[\mathbf{S}^{\prime}+\mathbf{Q}^{1 / 2}\right]=\mathbf{X}_{L} \Sigma \mathbf{X}_{R}$. Get $\hat{\Sigma}$ and $\mathbf{X}_{L}$ as in step 1.5

- Step 2.6 Compute fine-scale mean: $\boldsymbol{\mu}^{\prime}=\frac{1}{N_{e}} \sum_{i=1}^{N_{e}} \tilde{\mathbf{\Psi}}^{(i)}$.

- Step 2.7 Compute fine-scale deviations: $\mathbf{A}^{\prime \prime}=\left(\mathbf{b}^{(1)}, \mathbf{b}^{(2)}, \cdots, \mathbf{b}^{\left(N_{e}\right)}\right), \quad \mathbf{b}^{(i)}=\tilde{\boldsymbol{\Psi}}^{(i)}-\boldsymbol{\mu}$.

- Step 2.8 Update ensemble:

$$
\begin{aligned}
& \widehat{\mathbf{A}}=\left(\widehat{\boldsymbol{\Psi}}^{(1)}, \widehat{\boldsymbol{\Psi}}^{(2)}, \cdots, \widehat{\boldsymbol{\Psi}}^{\left(N_{e}\right)}\right), \\
& \widehat{\mathbf{A}}=\widetilde{\mathbf{A}}+\left(\mathbf{A}^{\prime \prime}\right)\left(\mathbf{S}^{\prime}\right)^{T} \hat{\mathbf{X}}_{L} \hat{\Sigma}-{ }^{2} \hat{\mathbf{X}}_{L}^{T} \mathbf{D}^{\prime} .
\end{aligned}
$$

\section{Remark 1:}

Note that steps 2.6 and 2.7 in above algorithm approximate the intermediate fine-scale error covariance

$$
\widetilde{\mathbf{P}^{f}} \approx \frac{1}{N_{e}-1} \mathbf{A}^{\prime \prime}\left(\mathbf{A}^{\prime \prime}\right)^{T}
$$

\section{Remark 2:}

Steps 2.1-2.3 accomplish ${ }^{3}$

$$
\mathbf{S}^{\prime}=\mathbf{U} \mathbf{A}^{\prime \prime} \text {. }
$$

Note that the above algorithm is independent of the choice of upscaling procedure and also, we can use the same algorithm for different kinds of coarse-scale observed data (if available).

\section{Remark 3:}

Note that the above coarse-scale constrained EnKF algorithm can be readily extended to incorporate data at multiple coarse scales, with appropriate upscaling procedure in $\mathbf{U}$. To elaborate, if we had another independent data at a scale different from $\mathbf{z}$, we use the estimates $\left(\left\{\widehat{\boldsymbol{\Psi}}^{(i)}\right\}_{i=1}^{N_{e}}\right.$.) obtained using $\mathbf{z}$, as intermediate solution, repeat Step 2 to assimilate the data at another scale. 